\title{
CONTINUOUS FUNCTIONS ON THE SPACE OF PROBABILITIES
}

\author{
S. C. BAGCHI AND B. V. RAO
}

\begin{abstract}
Weiss and Dubins discovered that any continuous function $g(P)$ on the space of probabilities $\mathscr{P}$ of a compact Hausdorff space $K$ is of the form $\int f d P^{\infty}$ for some continuous function $f$ on $K^{\infty}$. A short proof is given here.
\end{abstract}

Let $\mathscr{P}$ be the space of probabilities on the Borel $\sigma$ field of the unit interval $K=[0,1]$ equipped with the weak* topology so that $\mathscr{P}$ is again compact. It is easy to see that if $f$ is a real continuous function on $K^{\infty}$ then $g(P)=\int f d P^{\infty}$ is a continuous function on $\mathscr{P}$. Recently B. Weiss and L. E. Dubins [1] proved the converse. Namely,

THEOREM. Given a real continuous function $g$ on $\mathscr{P}$ there is a real continuous function $f$ on $K^{\infty}$ such that $g(P)=\int f d P^{\infty}$ for all $P$ in $\mathscr{P}$.

For $f \in C\left(K^{\infty}\right)$, let the function $g$ defined above be denoted by $T f . T$ is obviously a continuous linear operator on $C\left(K^{\infty}\right)$ into $C(\mathscr{P})$. Observe that if $f_{1}, f_{2}$ are in $C\left(K^{\infty}\right)$ then $f$ defined on $K^{\infty}$ by

$$
f\left(x_{1}, x_{2}, \ldots\right)=f_{1}\left(x_{1}, x_{3}, \ldots\right) f_{2}\left(x_{2}, x_{4}, \ldots\right)
$$

is continuous and $T f=T f_{1} \cdot T f_{2}$. As a consequence range of $T$ is a subalgebra of $C(\mathscr{P})$ containing constants and it clearly separates points. We shall now show that $T^{*}$ has closed range. This, by Banach's closed range theorem [2] implies that range of $T$ is closed and, hence, by the Stone-Weierstrass theorem, must be all of $C(\mathscr{P})$ as claimed.

Observe that $T^{*}: \mathfrak{M}(\mathscr{P}) \rightarrow \mathfrak{M}\left(K^{\infty}\right)$ is given by

$$
T^{*} \mu(A)=\int P^{\infty}(A) d \mu(P) \text { for } \mu \in \mathfrak{M}(\mathscr{P}) .
$$

$\mathfrak{M}(X)$ is the space of finite signed measures on $X$ with total variation norm. As $T$ has dense range, $T^{*}$ is one-to-one. If $\mu$ is positive then so is $T^{*} \mu$. If $\mu_{1}, \mu_{2}$ are positive and orthogonal then so are $T^{*} \mu_{1}$ and $T^{*} \mu_{2}$. Indeed, if $L \subset \mathscr{P}$ is a Borel set such that $\mu_{1}$ sits on $L$ and $\mu_{2}$ sits on $L^{c}$, then a simple application of the Glivenko-Cantelli Lemma implies that $T^{*} \mu_{1}$ sits on $A$ and $T^{*} \mu_{2}$ sits on $A^{c}$ where

$$
A=\left\{\left(x_{1}, x_{2}, \ldots\right) \in K^{\infty}: \frac{1}{n} \sum_{1}^{n} \delta_{x_{i}} \text { has a limit in } \mathscr{P} \text { and that limit } \in L\right\} .
$$

Received by the editors May 31, 1984.

1980 Mathematifs Subject Classification. Primary 60B99.

Key words and phrases. Weak* convergence of probabilities, closed range theorem.

(c)1985 American Mathematical Society $0002-9939 / 85 \$ 1.00+\$ .25$ per page 
As usual $\delta_{x}$ is the point mass at $x$. This implies that if $\mu=\mu^{+}-\mu^{-}$is the canonical Jordan Hahn decomposition of $\mu \in \mathfrak{M}(\mathscr{P})$ then $T^{*} \mu^{+}-T^{*} \mu^{-}$is the canonical decomposition of $T^{*} \mu$. This in turn implies that the total variations of $\mu$ and $T^{*} \mu$ are equal. In other words, $T^{*}$ is a norm preserving map and hence Range $T^{*}$ is closed. This completes the proof.

REMARK 1 . The same proof applies if $K$ is any compact Hausdorff space and $\mathscr{P}$ the probabilities on the Baire $\sigma$ field. This is the form proved in [1].

REmark 2. Dr. G. Jogesh Babu has yet another proof of the Theorem patterned after the original proof in [1] but more probabilistic in nature.

REMARK 3. The representation theorem of Hewitt-Savage for symmetric probabilities identifies range of $T^{*}$ as precisely the linear span of symmetric probabilities.

\section{REFERENCES}

1. L. E. Dubins, Bernstein like polynomial approximation in higher dimensions, Pacific J. Math 109 (1983), 305-311.

2. K. Yosida, Functional analysis, Springer-Verlag, Berlin and New York, 1974.

Statistics and Mathematics Division, Indian Statistical Institute, 203 Barrackpore Trunk Road, Calcutta 700 035, India 